\title{
On Smooth Mesoscopic Linear Statistics of the Eigenvalues of Random Permutation Matrices
}

\author{
Valentin Bahier ${ }^{1}$. Joseph Najnudel ${ }^{1}$
}

Received: 6 May 2020 / Revised: 26 April 2021 / Accepted: 12 May 2021 / Published online: 11 August 2021

(C) The Author(s) 2021

\begin{abstract}
We study the limiting behavior of smooth linear statistics of the spectrum of random permutation matrices in the mesoscopic regime, when the permutation follows one of the Ewens measures on the symmetric group. If we apply a smooth enough test function $f$ to all the determinations of the eigenangles of the permutations, we get a convergence in distribution when the order of the permutation tends to infinity. Two distinct kinds of limit appear: if $f(0) \neq 0$, we have a central limit theorem with a logarithmic variance; and if $f(0)=0$, the convergence holds without normalization and the limit involves a scale-invariant Poisson point process.
\end{abstract}

Keywords Random permutation matrices · Linear statistics of eigenvalues · Mesoscopic scale

Mathematics Subject Classification 15B52 - 60F05

\section{Notation}

In the present article, we denote by $A=\mathcal{O}(B)$ and by $A \ll B$ the fact that there exists a constant $C>0$ such that $|A| \leq C B$. The expressions $A=\mathcal{O}_{x, y}(B)$ and $A \ll_{x, y} B$ mean that there exists a quantity $C_{x, y}>0$ depending only on $x$ and $y$, such that $|A| \leq C_{x, y} B$.

\section{Introduction}

The spectrum of random permutation matrices has been studied with much attention in the last few decades. On the one hand, working with matrices gives a different way

\footnotetext{
Joseph Najnudel

joseph.najnudel@bristol.ac.uk

1 University of Bristol School of Mathematics, Bristol, United Kingdom 
to understand some of the classical properties satisfied by random permutations. On the other hand, the set of permutation matrices can be seen as a finite subgroup of the orthogonal group or the unitary group, and thus, an interesting problem consists in studying how similar are the spectral behaviors of random permutations and usual ensembles of random orthogonal or unitary matrices. For a random permutation matrix following one of the Ewens measures, the number of eigenvalues lying on a fixed arc of the unit circle has been studied in detail by Wieand [34] and satisfies a central limit theorem when the order $n$ goes to infinity, with a variance growing like $\log n$. This rate of growth is similar to what is obtained for the Circular Unitary Ensemble and random matrices on other compact groups, for which a central limit theorem also occurs, as it can be seen in Costin and Lebowitz [11], Soshnikov [30] and Wieand [33]. A similar result has recently been proved by Bahier [6], on the number of eigenvalues lying on a mesoscopic arc, for a suitable modification of Ewens distributed permutation matrices, and the growth of the variance is also the same as for the CUE, i.e., the logarithm of $n$ times the length of the interval. Some other results on the distribution of eigenvalues of matrices constructed from random permutations can be found in papers by Bahier [5], Evans [16], Najnudel and Nikeghbali [28], Tsou [31], Wieand [35].

The analogy between the permutation matrices and the CUE is not as strong when we consider smooth linear statistics of the eigenvalues. In this case, if we take a fixed, sufficiently smooth test function, it is known that the fluctuations of the corresponding linear statistics tend to a limiting distribution, without normalization, which is unusual for a limit theorem. In the CUE case, the distribution is Gaussian, as seen in Diaconis and Shahshahani [14], Johansson [22], Diaconis and Evans [13], and the variance is proportional to the squared $H^{1 / 2}$ norm of the test function. In the case of permutation matrices, the limiting distribution is not Gaussian anymore: its shape depends on the test function $f$ and can be explicitly described in terms of $f$ and a sequence of independent Poisson random variables. More detail can be found in Manstavicius [27], Ben Arous and Dang [7].

In the case of mesoscopic linear statistics, one also has a central limit theorem without normalization in the CUE case (see [29]). The behavior of mesoscopic linear statistics of other random matrix ensembles has also been studied: the Gaussian Unitary Ensemble (see [15]), more general Wigner matrices (see [19,20]) and determinantal processes (see [23]), the Circular Beta Ensemble (see [25]), the thinned CUE, for which a random subset of the eigenvalues has been removed (see [8]). However, the smooth mesoscopic linear statistics of permutation matrices have not been previously studied. The main point of the present article is to show that they also satisfy some limit theorems.

The precise framework is given as follows. We fix a parameter $\theta>0$, and we consider a sequence $\left(\sigma_{n}\right)_{n \geq 1}, \sigma_{n}$ following the the $\operatorname{Ewens}(\theta)$ distribution on the symmetric group $\mathfrak{S}_{n}$, that is to say

$$
\forall \sigma \in \mathfrak{S}_{n}, \mathbb{P}\left(\sigma_{n}=\sigma\right)=\mathbb{P}_{\theta}^{(n)}(\sigma)=\frac{\theta^{K(\sigma)}}{\theta(\theta+1) \cdots(\theta+n-1)},
$$

where $K(\sigma)$ denotes the total number of cycles of $\sigma$ once decomposed as a product of cycles with disjoint supports. Note that the particular case $\theta=1$ corresponds to the 
uniform distribution on $\mathfrak{S}_{n}$. The permutation matrix $M^{\sigma}$ associated with any element $\sigma$ of $\mathfrak{S}_{n}$ is defined as follows: for all $1 \leq i, j \leq n$,

$$
M_{i, j}^{\sigma}=\left\{\begin{array}{l}
1 \text { if } i=\sigma(j) \\
0 \text { otherwise. }
\end{array}\right.
$$

A key relationship between the cycle structure of $\sigma$ and the spectrum of the corresponding permutation matrix $M^{\sigma}$ appears in the expression of the characteristic polynomial of $M^{\sigma}$ :

$$
\forall x \in \mathbb{R}, \quad \operatorname{det}\left(I-x M^{\sigma}\right)=\prod_{j=1}^{n}\left(1-x^{j}\right)^{a_{j}^{\sigma}},
$$

where $a_{j}^{\sigma}$ denotes the number of $j$-cycles in the decomposition of $\sigma$ as a product of disjoint cycles. Indeed, $M^{\sigma}$ is similar to a block diagonal matrix where one adds one block equal to the companion matrix of $x^{j}-1$ for each $j$-cycle of $\sigma$. As a consequence, the cycle structure of $\sigma$ is fully determined by the spectrum of $M^{\sigma}$, counted with multiplicity.

In this paper, we are interested in the mesoscopic behavior of smooth linear statistics of the spectrum of $M^{\sigma_{n}}$ when $n$ goes to infinity. More precisely, we fix a function $f$ from $\mathbb{R}$ to $\mathbb{C}$ which satisfies the following regularity conditions:

$$
\left\{\begin{array}{l}
f \in \mathcal{C}^{2}(\mathbb{R}) \\
f^{\prime}, f^{\prime \prime} \in L^{1}(\mathbb{R}) \\
\exists M>0, \quad \exists \alpha>1, \quad \forall x \in \mathbb{R}, \quad|f(x)| \leq \frac{M}{(1+|x|)^{\alpha}} .
\end{array}\right.
$$

Moreover, we fix a sequence $\left(\delta_{n}\right)_{n \geq 1}$ in $\mathbb{R}_{+}^{*}=(0,+\infty)$ such that $\delta_{n} \rightarrow 0$ and $n \delta_{n} \rightarrow \infty$ when $n \rightarrow \infty$, which means that the corresponding scale is mesoscopic (small but large with respect to the average spacing between the eigenvalues of $M^{\sigma_{n}}$ ). In this article, we mainly study the following quantity:

$$
X_{\sigma_{n}, \delta_{n}}(f):=\sum_{x \in \mathbb{R}, e^{i x} \in S\left(\sigma_{n}\right)} m_{n}\left(e^{i x}\right) f\left(\frac{x}{2 \pi \delta_{n}}\right),
$$

where $S\left(\sigma_{n}\right)$ denotes the spectrum of $M^{\sigma_{n}}$ and $m_{n}\left(e^{i x}\right)$ is the multiplicity of $e^{i x}$ as an eigenvalue of $M^{\sigma_{n}}$. In other words, we sum the function $f$ at the eigenangles of $M^{\sigma_{n}}$, divided by $2 \pi \delta_{n}$ and counted with multiplicity. Notice that all the determinations of the eigenangles are considered here, and the set of $x$ involved in the sum is $2 \pi$ periodic. Notice that the sum giving $X_{\sigma_{n}, \delta_{n}}(f)$ is absolutely convergent, because of the assumption we make on the decay of $f$ at infinity. We will also consider the version of the linear statistics where we restrict the sum to the determinations of the eigenangles which are in the interval $(-\pi, \pi]$ :

$$
X_{\sigma_{n}, \delta_{n}}^{\prime}(f):=\sum_{x \in(\pi, \pi], e^{i x} \in S\left(\sigma_{n}\right)} m_{n}\left(e^{i x}\right) f\left(\frac{x}{2 \pi \delta_{n}}\right) .
$$


The two sums $X_{\sigma_{n}, \delta_{n}}(f)$ and $X_{\sigma_{n}, \delta_{n}}^{\prime}(f)$ can be both considered as natural: in $X_{\sigma_{n}, \delta_{n}}(f)$, the arbitrary choice of the determination of the eigenangles is avoided, since all determinations are considered, and then, the situation is more symmetric, whereas in $X_{\sigma_{n}, \delta_{n}}^{\prime}$, there is a bijection between eigenvalues and eigenangles, and considering the eigenangle in $(-\pi, \pi]$ is a standard choice.

If $f$ tends to zero sufficiently fast at infinity, it is natural to expect that $X_{\sigma_{n}, \delta_{n}}(f)$ and $X_{\sigma_{n}, \delta_{n}}^{\prime}(f)$ are close to each other and then have the same asymptotic behavior. Our main theorems provide precise results in this direction.

In order to state these theorems, we need to introduce the Fourier transform of $f$, normalized as follows:

$$
\hat{f}(\lambda):=\int_{\mathbb{R}} f(x) e^{-2 i \pi x \lambda} \mathrm{d} x,
$$

and the two following functions from $\mathbb{R}_{+}^{*}$ to $\mathbb{C}$ :

$$
\Theta_{f}: x \mapsto \sum_{k \in \mathbb{Z}} f(k x)
$$

and

$$
\Xi_{f}: x \mapsto \Theta_{f}(x)-f(0) \mathbb{1}_{x>1}-\frac{1}{x} \hat{f}(0) .
$$

The series defining $\Theta_{f}$ is absolutely convergent because of the assumptions (1). Our main result can now be stated as follows:

Theorem 1.1 Let $\left(\delta_{n}\right)_{n \geq 1}$ be a positive sequence such that $\delta_{n} \underset{n \rightarrow \infty}{\longrightarrow} 0$ and $n \delta_{n} \underset{n \rightarrow \infty}{\longrightarrow} \infty$, and let $f$ be a function from $\mathbb{R}$ to $\mathbb{C}$ satisfying the assumptions (1) given above.

(i) If $f(0) \neq 0$, then we have the following asymptotics:

$$
\mathbb{E}\left(X_{\sigma_{n}, \delta_{n}}(f)\right)=n \delta_{n} \hat{f}(0)-\theta \log \left(\delta_{n}\right) f(0)+\mathcal{O}_{f, \theta}(1)
$$

and

$$
\operatorname{Var}\left(X_{\sigma_{n}, \delta_{n}}(f)\right)=-\theta \log \left(\delta_{n}\right) f(0)^{2}+\mathcal{O}_{f, \theta}\left(\sqrt{-\log \left(\delta_{n}\right)}\right)
$$

Moreover, the following central limit theorem holds:

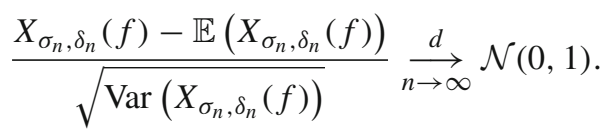

(ii) If $f(0)=0$, then we have the following convergence in distribution:

$$
X_{\sigma_{n}, \delta_{n}}(f)-n \delta_{n} \hat{f}(0) \underset{n \rightarrow \infty}{\stackrel{d}{\longrightarrow}} \sum_{y \in \mathcal{X}} \Xi_{f}(y),
$$


where $\mathcal{X}$ is a Poisson point process with intensity $\frac{\theta}{x} \mathrm{~d} x$ on $(0,+\infty)$ and where the sum on $\mathcal{X}$ in the right-hand side is a.s. absolutely convergent.

(iii) For any $\alpha>1$ such that (1) is satisfied, the results given in (i) and (ii) are still true if we replace $X_{\sigma_{n}, \delta_{n}}(f)$ by $X_{\sigma_{n}, \delta_{n}}^{\prime}(f)$, as soon as $\delta_{n}=o\left(n^{-1 / \alpha}\right)$ when $n \rightarrow \infty$.

Regarding $X_{\sigma_{n}, \delta_{n}}^{\prime}(f)$, we obtain a more refined version of point (iii) of Theorem 1.1. In order to state this result conveniently, let us introduce two additional notations: for all positive integers $j$ and all real numbers $x>0$,

$$
\Theta_{f, j}(x):=\sum_{k=\lfloor-j / 2\rfloor+1}^{\lfloor j / 2\rfloor} f(k x)
$$

and

$$
\Xi_{f, j}(x):=\Theta_{f, j}(x)-f(0) \mathbb{1}_{x>1}-\frac{1}{x} \hat{f}(0) .
$$

With this notation, all computations related to $X_{\sigma_{n}, \delta_{n}}^{\prime}$ are similar to the computations related to $X_{\sigma_{n}, \delta_{n}}$, except that $\Theta_{f}$ and $\Xi_{f}$ are replaced by $\Theta_{f, \ell}$ and $\Xi_{f, \ell}$ in the contribution of a cycle of length $\ell$.

Theorem 1.2 Assume (1), $f(0)=0$ and

$$
x \int_{|u|>x}\left|f^{\prime \prime}(u)\right| \mathrm{d} u \underset{x \rightarrow+\infty}{\longrightarrow} 0 .
$$

If $\left(\delta_{n}\right)_{n \geq 1}$ is a positive sequence such that $\delta_{n} \underset{n \rightarrow \infty}{\longrightarrow} 0, n \delta_{n} \underset{n \rightarrow \infty}{\longrightarrow} \infty$,

$$
n \delta_{n} \int_{|u|>\frac{1}{\delta_{n}}} f(u) \mathrm{d} u \underset{n \rightarrow \infty}{\longrightarrow} 0
$$

and

$$
\log (n) f\left( \pm \frac{1}{2 \delta_{n}}\right) \underset{n \rightarrow \infty}{\longrightarrow} 0
$$

then

$$
X_{\sigma_{n}, \delta_{n}}^{\prime}(f)-n \delta_{n} \hat{f}(0)=\sum_{\ell=1}^{n} a_{n, \ell} \Xi_{f, \ell}\left(\frac{1}{\ell \delta_{n}}\right) \underset{n \rightarrow \infty}{\stackrel{d}{\longrightarrow}} \sum_{y \in \mathcal{X}} \Xi_{f}(y),
$$

where $\mathcal{X}$ is a Poisson point process with intensity $\frac{\theta}{x} \mathrm{~d} x$ on $(0,+\infty)$. 
Example 1.3 - If $f \in \mathcal{C}_{c}^{2}(\mathbb{R})$ (i.e., $\mathcal{C}^{2}$ and compactly supported on $\mathbb{R}$ ) and $f(0)=0$, then all the conditions of Theorem 1.2 are satisfied, for every choice of $\delta_{n}$ such that $\delta_{n} \underset{n \rightarrow \infty}{\longrightarrow} 0$ and $n \delta_{n} \underset{n \rightarrow \infty}{\longrightarrow} \infty$.

- If $f$ satisfies (1), (3) and if $n \delta_{n}^{\alpha} \rightarrow 0$ (it is in particular the case if $\delta_{n}=n^{-\varepsilon}$ for any $\varepsilon \in\left(\frac{1}{\alpha}, 1\right)$ ), then all the conditions of Theorem 1.2 are satisfied. Indeed,

$$
\left|n \delta_{n} \int_{|u|>\frac{1}{\delta_{n}}} f(u) \mathrm{d} u\right| \leq n \delta_{n} \int_{|u|>\frac{1}{\delta_{n}}} \frac{M}{(1+|u|)^{\alpha}} \mathrm{d} u \ll n \delta_{n}^{\alpha}
$$

and

$$
\left|\log (n) f\left( \pm \frac{1}{2 \delta_{n}}\right)\right| \ll \log (n) \delta_{n}^{\alpha}=o\left(n \delta_{n}^{\alpha}\right)
$$

- If $f \in \mathcal{S}(\mathbb{R})$ (i.e. in the Schwartz space of $\mathbb{R}$ ), and if $\delta_{n}=n^{-\varepsilon}$ for any $\varepsilon \in(0,1)$, then all the conditions of Theorem 1.2 are satisfied.

The assumptions (1) of Theorem 1.1 are made in order to apply Proposition 1 and Lemma 1: we do not expect that our assumptions are optimal, but it is necessary to have some properties of regularity and decay of $f$. The extra assumptions in Theorem 1.2 are used in order to compare $X_{\sigma_{n}, \delta_{n}}^{\prime}(f)$ and $X_{\sigma_{n}, \delta_{n}}(f)$ : it may also be possible that they can be relaxed, but they cannot be totally removed, as shown in the following counterexamples.

Counterexample 1.4 - If $f(x)=1 /(1+|x|)$, and if $\delta_{n}$ is such that $\delta_{n} \underset{n \rightarrow \infty}{\longrightarrow} 0$ and $n \delta_{n} \underset{n \rightarrow \infty}{\longrightarrow} \infty$, then $X_{\sigma_{n}, \delta_{n}}(f)$ is infinite, whereas in the expression of $X_{\sigma_{n}, \delta_{n}}^{\prime}(f), a$ cycle of length $\ell$ gives a contribution of

$$
\begin{aligned}
\sum_{k=\lfloor-\ell / 2\rfloor+1}^{\lfloor\ell / 2\rfloor} \frac{1}{1+|k| /\left(\ell \delta_{n}\right)} & =\mathcal{O}(1)+2 \sum_{k=1}^{\lfloor\ell / 2\rfloor} \frac{\ell \delta_{n}}{\ell \delta_{n}+k} \\
& =2 \ell \delta_{n}\left(\log \left(\frac{\ell \delta_{n}+\ell / 2}{\ell \delta_{n}+1}\right)+\mathcal{O}(1)\right)+\mathcal{O}(1) \\
& =2 \ell \delta_{n}\left(\log \ell-\log \left(1+\ell \delta_{n}\right)+\mathcal{O}(1)\right)+\mathcal{O}(1)
\end{aligned}
$$

If $\ell \geq \delta_{n}^{-1}$, we get an estimate:

$$
2 \ell \delta_{n}\left(\log \ell-\log \left(\ell \delta_{n}\right)+\mathcal{O}(1)\right)+\mathcal{O}(1)=2 \ell \delta_{n} \log \left(\delta_{n}^{-1}\right)+\mathcal{O}\left(\ell \delta_{n}\right),
$$

and if $\ell \leq \delta_{n}^{-1}$, we get

$$
2 \ell \delta_{n}(\log \ell+\mathcal{O}(1))+\mathcal{O}(1)=2 \ell \delta_{n} \log \ell+\mathcal{O}(1) .
$$

By the Feller coupling described in Section 3 (see the proof of Lemma 2), if $a_{n, \ell}$ is the number of $\ell$-cycles in $\sigma_{n}$, then the variables $\left(a_{n, \ell}\right)_{1 \leq \ell \leq n}$ can be coupled 
with independent Poisson variables $\left(W_{\ell}\right)_{1 \leq \ell \leq n}$, where $W_{\ell}$ has parameter $\theta / \ell$, in such a way that $a_{n, \ell} \leq W_{\ell}$ for all $\ell \in\{1, \ldots, n\}$, except for at most one value of $\ell$ for which $a_{n, \ell}=W_{\ell}+1$. Hence, the number of cycles smaller than or equal to $\delta_{n}^{-1}$ has expectation at most $\theta\left(1+\log \left(\delta_{n}^{-1}\right)\right)$ and the sum of their lengths has expectation at most $1+\theta \delta_{n}^{-1}$. Hence, as soon as $\omega(n) \geq 1$ goes to infinity at infinity, the number of small cycles is at most $\omega(n) \theta\left(1+\log \left(\delta_{n}^{-1}\right)\right)$, and the sum of their length is at most $\omega(n)\left(1+\theta \delta_{n}^{-1}\right)$, with probability tending to 1 when $n \rightarrow \infty$. If we take $\omega(n)$ going to infinity slower than $n \delta_{n}$, we deduce that with probability going to 1 , the sum of the lengths of the cycles larger than $\delta_{n}^{-1}$ is $n-o(n)$ with probability tending to 1 . Hence, their contribution in $X_{\sigma_{n}, \delta_{n}}^{\prime}(f)$ is equivalent to $2 n \delta_{n} \log \left(\delta_{n}^{-1}\right)$. The contribution of the cycles smaller than $\delta_{n}^{-1}$ is dominated by $\delta_{n} \log \left(\delta_{n}^{-1}\right)$ times the sum of their lengths, plus the number of these cycles. With probability going to 1 , this contribution is dominated by

$$
\left(\delta_{n} \log \left(\delta_{n}^{-1}\right)\left(1+\delta_{n}^{-1}\right)+1+\log \left(\delta_{n}^{-1}\right)\right) \omega(n) \ll \log \left(\delta_{n}^{-1}\right) \omega(n)
$$

if $n$ is large enough, and if $\omega(n)$ goes to infinity slower than $n \delta_{n}$, we deduce that

$$
X_{\sigma_{n}, \delta_{n}}^{\prime}(f)=(2+o(1)) n \delta_{n} \log \left(\delta_{n}^{-1}\right)
$$

with probability tending to 1 when $n \rightarrow \infty$. This behavior at infinity does not correspond to what we get in the theorems.

- If $f(x)=x^{2} /\left(1+x^{4}\right)$, $f$ satisfies the assumptions of Theorem 1.1 (ii), and then,

$$
X_{\sigma_{n}, \delta_{n}}-n \delta_{n} \hat{f}(0) \underset{n \rightarrow \infty}{\stackrel{d}{\longrightarrow}} \sum_{y \in \mathcal{X}} \Xi_{f}(y) \text {. }
$$

If we replace $X_{\sigma_{n}, \delta_{n}}$ by $X_{\sigma_{n}, \delta_{n}}^{\prime}$, then we subtract at least $n$ terms of the form $f\left(x / 2 \pi \delta_{n}\right)$ for $\pi<x \leq 3 \pi$ and then at least a quantity of order $n \delta_{n}^{2}$. If $n \delta_{n}^{2}$ tends to infinity when $n \rightarrow \infty$ (for example if $\left.\delta_{n}=n^{-1 / 3}\right)$, then

$$
X_{\sigma_{n}, \delta_{n}}^{\prime}-n \delta_{n} \hat{f}(0) \underset{n \rightarrow \infty}{\stackrel{d}{\longrightarrow}}-\infty
$$

in the sense that

$$
\mathbb{P}\left[X_{\sigma_{n}, \delta_{n}}^{\prime}-n \delta_{n} \hat{f}(0)>-A\right] \underset{n \rightarrow \infty}{\longrightarrow} 0
$$

for any fixed $A>0$. Hence, Theorem 1.1 (ii) does not extend to $X_{\sigma_{n}, \delta_{n}}^{\prime}$.

\section{Outline of the Paper}

In Section 2, we give an expression of $X_{\sigma_{n}, \delta_{n}}(f)$ and provide preliminary results involving the regularity of $f$. In Section 3 , we recall the construction of the Feller coupling, which is a suitable feature for dealing with numbers of cycles of equal 
size in random permutations. The Feller coupling provides a comparison between $X_{\sigma_{n}, \delta_{n}}(f)$ and a sum involving independent Poisson random variables. In Section 4, we combine this comparison and a central limit theorem in order to show the first part of Theorem 1.1. In Section 5, a comparison between the Fourier transforms of $X_{\sigma_{n}, \delta_{n}}(f)$ and the sum involving Poisson variables is then combined with the convergence of this sum toward the right-hand side of (2) in order to prove the second part of Theorem 1.1. The third part of Theorem 1.1 is then deduced by showing that $X_{\sigma_{n}, \delta_{n}}(f)$ and $X_{\sigma_{n}, \delta_{n}}^{\prime}(f)$ are close to each other under the assumptions which are considered. Theorem 1.2 is proved in Section 6.

\section{Expression of $X_{\sigma_{n}, \delta_{n}}(f)$ in Terms of $\Theta_{f}$ and $\Xi_{f}$}

In the spectrum of $M^{\sigma_{n}}$, each cycle of length $\ell$ gives eigenangles equal to all multiples of $2 \pi / \ell$. The contribution of these eigenangles in the sum $X_{\sigma_{n}, \delta_{n}}(f)$ is:

$$
\sum_{k \in \mathbb{Z}} f\left(\frac{k}{\ell \delta_{n}}\right)=\Theta_{f}\left(\frac{1}{\ell \delta_{n}}\right)
$$

Then, if we denote by $a_{n, j}$ the number of $j$-cycles in the decomposition of $\sigma_{n}$ as a product of cycles with disjoint support, we get:

$$
\begin{aligned}
X_{\sigma_{n}, \delta_{n}}(f) & =\sum_{\ell=1}^{n} a_{n, \ell} \Theta_{f}\left(\frac{1}{\ell \delta_{n}}\right) \\
& =\sum_{\ell=1}^{n} a_{n, \ell} \Xi_{f}\left(\frac{1}{\ell \delta_{n}}\right)+f(0) \sum_{\ell<\delta_{n}^{-1}} a_{n, \ell}+\hat{f}(0) \sum_{\ell=1}^{n} \ell \delta_{n} a_{n, \ell} .
\end{aligned}
$$

Since the total number of elements of all cycles is $n$, we have

$$
\sum_{\ell=1}^{n} \ell a_{n, \ell}=n
$$

and then,

$$
X_{\sigma_{n}, \delta_{n}}(f)=n \delta_{n} \hat{f}(0)+\sum_{\ell=1}^{n} a_{n, \ell} \Xi_{f}\left(\frac{1}{\ell \delta_{n}}\right)+f(0) \sum_{\ell<\delta_{n}^{-1}} a_{n, \ell}
$$

Note that $\hat{f}(0)$ is the integral of $f$, and then, the term $n \delta_{n} \hat{f}(0)$ is what we would expect if the $n$ eigenangles lying in $[0,2 \pi)$ were uniformly distributed. 
First note that under (1), the Poisson summation formula applies and gives for all $x>0$,

$$
\Theta_{f}(x)=\frac{1}{x} \Theta_{\hat{f}}\left(\frac{1}{x}\right) .
$$

We now get the following asymptotic result on $\Theta_{f}$ :

Proposition 1 Assume (1). Then,

(i) $\Theta_{f}$ is continuous on $\mathbb{R}_{+}^{*}$ and converges at infinity to $f(0)$ with rate dominated by $\frac{1}{x^{\alpha}}$ (where $\alpha$ is given by (1)).

(ii) $\Theta_{\hat{f}}$ is continuous on $\mathbb{R}_{+}^{*}$ and converges at infinity to $\hat{f}(0)$ with rate dominated by $\frac{1}{x^{2}}$.

Proof We prove the two items separately.

- Proof of (i) Since $f$ is assumed to be continuous, the functions $f_{k}: x \mapsto f(k x)$ are clearly continuous on $(0,+\infty)$ for all $k \in \mathbb{Z}$. Moreover, for all $k \in \mathbb{Z} \backslash\{0\}$ and for all $x$ in any compact set $[A, B] \subset(0,+\infty)$,

$$
\left|f_{k}(x)\right| \leq \frac{M}{\left(1+|k x|^{\alpha}\right)} \leq \frac{M}{|k|^{\alpha} A^{\alpha}} ;
$$

hence, $\sum_{k} f_{k}$ converges uniformly on compact sets of $(0,+\infty)$. We deduce the continuity of $\Theta_{f}$. For the convergence of $\Theta_{f}$ to $f(0)$ at infinity, we only have to notice that for all $x \geq 1$,

$$
\sum_{k \neq 0}|f(k x)| \leq \frac{M}{x^{\alpha}} \sum_{k \neq 0} \frac{1}{|k|^{\alpha}} \ll_{M, \alpha} \frac{1}{x^{\alpha}} .
$$

- Proof of (ii) It is clear that the functions $g_{k}: x \mapsto \hat{f}(k x)$ are continuous on $(0,+\infty)$ for all $k \in \mathbb{Z}$, and from two consecutive integrations by parts, it follows that for all $k \in \mathbb{Z} \backslash\{0\}$ and for all $x$ in any compact set $[A, B] \subset(0,+\infty)$,

$$
\begin{aligned}
\left|g_{k}(x)\right| & =\left|\frac{1}{(2 i \pi k x)^{2}} \int_{-\infty}^{+\infty} f^{\prime \prime}(y) \mathrm{e}^{-2 i \pi k x y} \mathrm{~d} y\right| \\
& \leq \frac{1}{4 \pi^{2} k^{2} A^{2}} \int_{-\infty}^{+\infty}\left|f^{\prime \prime}(y)\right| \mathrm{d} y
\end{aligned}
$$

hence, $\sum_{k} g_{k}$ converges uniformly on compact sets of $(0,+\infty)$. Note that there is no boundary term in the integration by parts, since by assumption, $f$ goes to zero at infinity, and $f^{\prime}$ and $f^{\prime \prime}$ are integrable, which implies that $f^{\prime}$ also goes to zero at infinity. 
Now, for all $x \geq 1$,

$$
\sum_{k \neq 0}|\hat{f}(k x)| \leq \frac{1}{x^{2}} \times \frac{1}{4 \pi^{2}} \int_{-\infty}^{+\infty}\left|f^{\prime \prime}(y)\right| \mathrm{d} y \sum_{k \neq 0} \frac{1}{k^{2}} \ll \frac{1}{x^{2}},
$$

and the proof is complete.

From the proposition just above, we deduce the following lemma:

Lemma 1 If the function $f$ satisfies the assumptions (1), then for all $x \in \mathbb{R}_{+}^{*}$,

$$
\left|\Xi_{f}(x)\right| \ll_{f} \min (x, 1 / x) .
$$

In particular,

$$
\int_{0}^{\infty} \frac{\left|\Xi_{f}(x)\right|}{x} d x<\infty
$$

Moreover, $\Xi_{f}(x)$ is continuous at any point of $\mathbb{R}_{+}^{*} \backslash\{1\}$, and also at 1 if $f(0)=0$.

Proof We have, for all $x \in(0,1]$,

$$
\left|\Xi_{f}(x)\right|=\left|\Theta_{f}(x)-\frac{1}{x} \hat{f}(0)\right|=\frac{1}{x}\left|\Theta_{\hat{f}}\left(\frac{1}{x}\right)-\hat{f}(0)\right| \ll_{f} \frac{1}{x} \times x^{2}=x,
$$

and for all $x \in(1, \infty)$,

$$
\left|\Xi_{f}(x)\right| \leq\left|\Theta_{f}(x)-f(0)\right|+\frac{1}{x}|\hat{f}(0)| \ll_{f, \alpha} \frac{1}{x^{\alpha}}+\frac{1}{x} \ll \frac{1}{x} .
$$

The continuity of $\Xi_{f}$ is an immediate consequence of the continuity of $\Theta_{f}$.

\section{The Feller Coupling}

In [18], Feller introduces a construction of a uniform permutation on the symmetric group, such that the cycle lengths are given by the spacings between successes in independent Bernoulli trials. This construction can be extended to general Ewens distributions and provides a coupling between the cycle counts of a random permutation and a sequence of independent Poisson random variables. The coupling procedure and many related results are proved in [2] and [7, Section 4]: in order to make this article self-contained, we reproduce here some of the discussion given in [7, Section 4]. We consider a sequence $\left(\xi_{i}\right)_{i \geq 1}$ of independent Bernoulli random variables, such that

$$
\mathbb{P}\left[\xi_{i}=1\right]=\frac{\theta}{\theta+i-1}, \quad \mathbb{P}\left[\xi_{i}=0\right]=\frac{i-1}{\theta+i-1} .
$$


For $1 \leq \ell \leq n$, let us denote the number of spacings of length $\ell$ in the sequence $1 \xi_{2} \xi_{3} \ldots \xi_{n} 1$ by $c_{\ell}(n)$, i.e.:

$$
c_{\ell}(n)=\sum_{i=1}^{n-\ell} \xi_{i}\left(1-\xi_{i+1}\right) \ldots\left(1-\xi_{i+\ell-1}\right) \xi_{i+\ell}+\xi_{n-\ell+1}\left(1-\xi_{n-\ell+2}\right) \ldots\left(1-\xi_{n}\right) .
$$

Define

$$
W_{\ell}:=\sum_{i=1}^{\infty} \xi_{i}\left(1-\xi_{i+1}\right) \ldots\left(1-\xi_{i+\ell-1}\right) \xi_{i+\ell} .
$$

The following result is proved in [7, Theorem 4.1]):

Proposition 2 (i) $\left(c_{\ell}(n)\right)_{1 \leq \ell \leq n}$ has the same probability distribution as $\left(a_{n, \ell}\right)_{1 \leq \ell \leq n}$, where $a_{n, \ell}$ denotes the number of $\ell$-cycles in the random permutation $\sigma_{n}$.

(ii) $\left(W_{\ell}\right)_{\ell \geq 1}$ are independent Poisson random variables, with $\mathbb{E}\left[W_{\ell}\right]=\theta / \ell$.

We deduce the following lemma:

Lemma 2 For all $n \geq 1$, one can couple the numbers $a_{n, \ell}$ of $\ell$-cycles in the random permutation $\sigma_{n}$, with a sequence of independent Poisson variables $W_{\ell}$ of parameters $\theta / \ell$, in such a way that

$$
\mathbb{E}\left(\left(\sum_{\ell \leq n}\left|a_{n, \ell}-W_{\ell}\right|\right)^{2}\right) \leq C(\theta),
$$

where $C(\theta)$ is a constant which does not depend on $n$.

Proof We choose a coupling such that that $a_{n, \ell}=c_{\ell}(n)$, which is possible by Proposition 2 . We then have $a_{n, \ell}=c_{\ell}(n) \leq W_{\ell}$, except for at most one value of $\ell$, for which $a_{n, \ell}$ may be equal to $W_{\ell}+1$. Hence,

$$
\sum_{\ell \leq n}\left|a_{n, \ell}-W_{\ell}\right| \leq 2+\sum_{\ell \leq n}\left(W_{\ell}-a_{n, \ell}\right)
$$

It is then enough to bound the $L^{2}$ norm of $G_{n}-H_{n}$ by a quantity depending only on $\theta$, for $G_{n}:=\sum_{j=1}^{n} a_{n, j}, H_{n}:=\sum_{j=1}^{n} W_{j}$. Such a bound is a consequence of [7, Lemma 4.8], in the case where $u_{j}=1$ for $1 \leq j \leq n$.

The lemma proved here allows to compare the quantity $X_{\sigma_{n}, \delta_{n}}(f)$ with a linear combination of independent Poisson random variables, for which classical tools in probability theory can be used to prove limit theorems. 


\section{Proof of Theorem 1.1 (i)}

We couple the variables $\left(a_{n, \ell}\right)_{1 \leq \ell \leq n}$ with independent Poisson variables $\left(W_{\ell}\right)_{\ell \geq 1}$ by using the Feller coupling, as in the previous section. From (4), we get

$$
\begin{aligned}
X_{\sigma_{n}, \delta_{n}}(f)= & n \delta_{n} \hat{f}(0)+f(0) \sum_{\ell<\delta_{n}^{-1}} W_{\ell}+f(0) \sum_{\ell<\delta_{n}^{-1}}\left(a_{n, \ell}-W_{\ell}\right) \\
& +\sum_{\ell=1}^{n} a_{n, \ell} \Xi_{f}\left(\frac{1}{\ell \delta_{n}}\right) .
\end{aligned}
$$

In order to prove the first part of Theorem 1.1, we will show that the sum of the two first terms satisfies the same central limit theorem and that the two last terms are bounded in $L^{2}$. We first prove the following result:

Proposition 3 We have:

$$
\begin{aligned}
& \mathbb{E}\left[n \delta_{n} \hat{f}(0)+f(0) \sum_{\ell<\delta_{n}^{-1}} W_{\ell}\right]=n \delta_{n} \hat{f}(0)-\theta \log \left(\delta_{n}\right) f(0)+\mathcal{O}_{f, \theta}(1), \\
& \operatorname{Var}\left[n \delta_{n} \hat{f}(0)+f(0) \sum_{\ell<\delta_{n}^{-1}} W_{\ell}\right]=-\theta \log \left(\delta_{n}\right) f(0)^{2}+\mathcal{O}_{f, \theta}(1),
\end{aligned}
$$

and

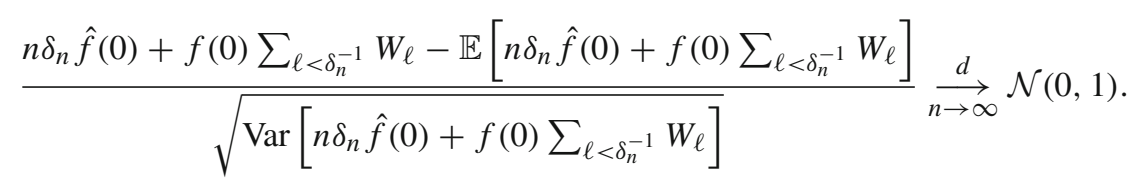

Proof Since $\left(W_{\ell}\right)_{\ell \geq 1}$ are independent Poisson random variables, $W_{\ell}$ with parameter $\theta / \ell$, we get

$$
\mathbb{E}\left(\sum_{\ell<\delta_{n}^{-1}} W_{\ell}\right)=\operatorname{Var}\left(\sum_{\ell<\delta_{n}^{-1}} W_{\ell}\right)=\sum_{\ell<\delta_{n}^{-1}} \frac{\theta}{\ell}=\theta \log \left(\delta_{n}^{-1}\right)+\mathcal{O}_{\theta}(1)
$$

which gives the estimates of the proposition for the expectation and the variance. The central limit theorem is easily obtained by applying the Lindeberg-Feller criterion, since the variables $\left(W_{\ell}\right)_{\ell \geq 1}$ are independent.

We then prove that the two last terms of (6) are bounded in $L^{2}$ : 
Proposition 4 We have the estimate:

$$
\mathbb{E}\left[\left(f(0) \sum_{\ell<\delta_{n}^{-1}}\left|a_{n, \ell}-W_{\ell}\right|+\sum_{\ell=1}^{n} a_{n, \ell}\left|\Xi_{f}\left(\frac{1}{\ell \delta_{n}}\right)\right|\right)^{2}\right]=\mathcal{O}_{f, \theta}(1)
$$

Proof By Lemma 2, it is enough to show

$$
\mathbb{E}\left[\left(\sum_{\ell=1}^{n} a_{n, \ell}\left|\Xi_{f}\left(\frac{1}{\ell \delta_{n}}\right)\right|\right)^{2}\right]=\mathcal{O}_{f, \theta}(1)
$$

Moreover, we have $a_{n, \ell} \leq W_{\ell}$ for all $\ell$ except at most one value, for which we may have $a_{n, \ell}=W_{\ell}+1$. It is then enough to check

$$
\mathbb{E}\left[\left(\sup _{\mathbb{R}_{+}^{*}}\left|\Xi_{f}\right|+\sum_{\ell=1}^{n} W_{\ell}\left|\Xi_{f}\left(\frac{1}{\ell \delta_{n}}\right)\right|\right)^{2}\right]=\mathcal{O}_{f, \theta}(1)
$$

or equivalently,

$$
\mathbb{E}\left[\sup _{\mathbb{R}_{+}^{*}}\left|\Xi_{f}\right|+\sum_{\ell=1}^{n} W_{\ell}\left|\Xi_{f}\left(\frac{1}{\ell \delta_{n}}\right)\right|\right]=\mathcal{O}_{f, \theta}(1)
$$

and

$$
\operatorname{Var}\left(\sum_{\ell=1}^{n} W_{\ell}\left|\Xi_{f}\left(\frac{1}{\ell \delta_{n}}\right)\right|\right)=\mathcal{O}_{f, \theta}(1)
$$

These estimates are implied by the estimate

$$
\sup _{\mathbb{R}_{+}^{*}}\left|\Xi_{f}\right|+\sum_{\ell=1}^{n} \frac{1}{\ell}\left(\left|\Xi_{f}\left(\frac{1}{\ell \delta_{n}}\right)\right|+\left|\Xi_{f}\left(\frac{1}{\ell \delta_{n}}\right)\right|^{2}\right)=\mathcal{O}_{f}(1),
$$

which is a direct consequence of Lemma 1.

It is now easy to deduce Theorem 1.1 (i) from the two propositions just above. The estimate of the expectation is immediate, and the estimate of the variance is directly deduced from the fact that

$$
\operatorname{Var}(A+B)=\operatorname{Var}(A)+\operatorname{Var}(B)+\mathcal{O}\left(\operatorname{Var}^{1 / 2}(A) \operatorname{Var}^{1 / 2}(B)\right)
$$


For the central limit theorem, we know that

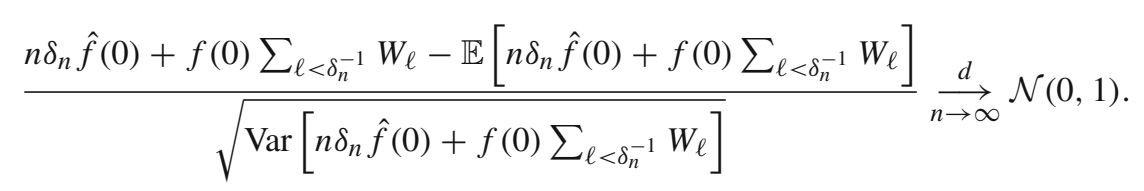

and

$$
\frac{f(0) \sum_{\ell<\delta_{n}^{-1}}\left(a_{n, \ell}-W_{\ell}\right)+\sum_{\ell=1}^{n} a_{n, \ell} \Xi_{f}\left(\frac{1}{\ell \delta_{n}}\right)}{\sqrt{\operatorname{Var}\left[n \delta_{n} \hat{f}(0)+f(0) \sum_{\ell<\delta_{n}^{-1}} W_{\ell}\right]}} \underset{n \rightarrow \infty}{\longrightarrow} 0
$$

in $L^{2}$, since the numerator is bounded in $L^{2}$ and the denominator tends to infinity with $n$. Hence,

$$
\frac{\mathbb{E}\left[f(0) \sum_{\ell<\delta_{n}^{-1}}\left(a_{n, \ell}-W_{\ell}\right)+\sum_{\ell=1}^{n} a_{n, \ell} \Xi_{f}\left(\frac{1}{\ell \delta_{n}}\right)\right]}{\sqrt{\operatorname{Var}\left[n \delta_{n} \hat{f}(0)+f(0) \sum_{\ell<\delta_{n}^{-1}} W_{\ell}\right]}} \underset{n \rightarrow \infty}{\longrightarrow} 0
$$

and by Slutsky’s lemma,

$$
\frac{X_{\sigma_{n}, \delta_{n}}-\mathbb{E}\left[X_{\sigma_{n}, \delta_{n}}\right]}{\sqrt{\operatorname{Var}\left[n \delta_{n} \hat{f}(0)+f(0) \sum_{\ell<\delta_{n}^{-1}} W_{\ell}\right]}} \stackrel{d}{\stackrel{\leftrightarrow}{\longrightarrow}} \mathcal{N}(0,1) .
$$

Since the variance estimates we know imply that

$$
\frac{\sqrt{\operatorname{Var}\left(n \delta_{n} \hat{f}(0)+f(0) \sum_{\ell<\delta_{n}^{-1}} W_{\ell}\right)}}{\sqrt{\operatorname{Var}\left(X_{\sigma_{n}, \delta_{n}}\right)}} \underset{n \rightarrow \infty}{\longrightarrow} 1 \text {, }
$$

we are done.

\section{Proof of Theorem 1.1 (ii) and (iii)}

Let $A_{n}:=\sum_{\ell=1}^{n} a_{n, \ell} \Xi_{f}\left(\frac{1}{\ell \delta_{n}}\right), B_{n}:=\sum_{\ell=1}^{n} W_{\ell} \Xi_{f}\left(\frac{1}{\ell \delta_{n}}\right)$ and $Z=\sum_{y \in \mathcal{X}} \Xi_{f}(y)$. Here, $a_{n, \ell}$ and $W_{\ell}$ are again related by the Feller coupling. Notice that the sum defining $Z$ is a.s. absolutely convergent, since

$$
\mathbb{E}\left[\sum_{y \in \mathcal{X}}\left|\Xi_{f}(y)\right|\right]=\theta \int_{0}^{\infty}\left|\Xi_{f}(x)\right| \frac{d x}{x}<\infty
$$


by Lemma 1 .

We are going to prove the result in two steps:

(i) For all $t \in \mathbb{R}, \mathbb{E}\left(\mathrm{e}^{i t B_{n}}\right) \underset{n \rightarrow \infty}{\longrightarrow} \mathbb{E}\left(\mathrm{e}^{i t Z}\right)$.

(ii) For all $t \in \mathbb{R},\left|\mathbb{E}\left(\mathrm{e}^{i t A_{n}}\right)-\mathbb{E}\left(\mathrm{e}^{i t B_{n}}\right)\right|_{n \rightarrow \infty} 0$.

(i): Let $t \in \mathbb{R}$. Using that the variables $W_{j}$ are independent,

$$
\begin{aligned}
\mathbb{E}\left(\mathrm{e}^{i t B_{n}}\right) & =\prod_{\ell=1}^{n} \mathbb{E}\left(\mathrm{e}^{i t W_{\ell} \Xi_{f}\left(1 / \ell \delta_{n}\right)}\right) \\
& =\prod_{\ell=1}^{n} \exp \left(\frac{\theta}{\ell}\left(\mathrm{e}^{i t \Xi_{f}\left(1 / \ell \delta_{n}\right)}-1\right)\right) \\
& =\exp \left(\theta \delta_{n} \sum_{\ell=1}^{n} \frac{1}{\ell \delta_{n}}\left(\mathrm{e}^{i t \Xi_{f}\left(1 / \ell \delta_{n}\right)}-1\right)\right) \\
& =\exp \left(\theta \delta_{n} \sum_{1 \leq \ell \leq n, \ell \delta_{n} \in[1 / R, R]} \frac{1}{\ell \delta_{n}}\left(\mathrm{e}^{i t \Xi_{f}\left(1 / \ell \delta_{n}\right)}-1\right)\right) \\
& \times \exp \left(\theta \delta_{n} \sum_{1 \leq \ell \leq n, \ell \delta_{n} \notin[1 / R, R]} \frac{1}{\ell \delta_{n}}\left(\mathrm{e}^{i t \Xi_{f}\left(1 / \ell \delta_{n}\right)}-1\right)\right),
\end{aligned}
$$

for any $R>1$. For fixed $R$ and $n$ large enough depending on $R$, the condition $1 \leq \ell \leq n$ can be discarded in the first exponential of the last product, since $\delta_{n} \rightarrow 0$ and $n \delta_{n} \rightarrow \infty$ when $n \rightarrow \infty$. The sum in the first exponential is then a Riemann sum, which by continuity of $\Xi_{f}$ (proved in Lemma 1: recall that $f(0)=0$ in this section), shows that the exponential tends to

$$
\exp \left(\theta \int_{1 / R}^{R} \frac{1}{x}\left(\mathrm{e}^{i t \Xi_{f}(1 / x)}-1\right) \mathrm{d} x\right)
$$

when $n$ goes to infinity. On the other hand, by Lemma 1 the sum inside the second exponential is dominated by

$$
\sum_{\ell \delta_{n}>R} \frac{1}{\left(\ell \delta_{n}\right)^{2}}+\sum_{\ell \delta_{n}<1 / R} 1=\mathcal{O}\left(\left(R \delta_{n}\right)^{-1}\right),
$$

and then the second exponential is

$$
\exp \left(\mathcal{O}_{f, \theta, t}(1 / R)\right)=1+\mathcal{O}_{f, \theta, t}(1 / R)
$$


Now, if $L$ is the limit of a subsequence $\left(\mathbb{E}\left(\mathrm{e}^{i t B_{n_{k}}}\right)\right)_{k \geq 1}$, then

$$
\begin{aligned}
& \exp \left(\theta \delta_{n_{k}} \sum_{1 \leq \ell \leq n_{k}, \ell \delta_{n_{k}} \notin[1 / R, R]} \frac{1}{\ell \delta_{n_{k}}}\left(\mathrm{e}^{i t \Xi_{f}\left(1 / \ell \delta_{n_{k}}\right)}-1\right)\right) \\
& \underset{k \rightarrow \infty}{\longrightarrow} L \exp \left(-\theta \int_{1 / R}^{R} \frac{1}{x}\left(\mathrm{e}^{i t \Xi_{f}(1 / x)}-1\right) \mathrm{d} x\right) .
\end{aligned}
$$

Since the left-hand side of the convergence is $1+\mathcal{O}_{f, \theta, t}(1 / R)$, we deduce that

$$
L=\left(1+\mathcal{O}_{f, \theta, t}(1 / R)\right) \exp \left(\theta \int_{1 / R}^{R} \frac{1}{x}\left(\mathrm{e}^{i t \Xi_{f}(1 / x)}-1\right) \mathrm{d} x\right)
$$

Letting $R \rightarrow \infty$, we deduce

$$
L=\exp \left(\theta \int_{0}^{\infty} \frac{1}{x}\left(\mathrm{e}^{i t \Xi_{f}(1 / x)}-1\right) \mathrm{d} x\right)=\exp \left(\theta \int_{0}^{\infty} \frac{1}{y}\left(\mathrm{e}^{i t \Xi_{f}(y)}-1\right) \mathrm{d} y\right),
$$

where the convergence of the integrals is insured by the integrability of $\left|\Xi_{f}(x)\right| d x / x$ given in Lemma 1. By Campbell's theorem,

$$
L=\mathbb{E}\left(\mathrm{e}^{i t Z}\right)
$$

i.e., $\mathbb{E}\left(\mathrm{e}^{i t Z}\right)$ is the unique possible limit of a subsequence of $\left(\mathbb{E}\left(\mathrm{e}^{i t B_{n}}\right)\right)_{n \geq 1}$. Since this sequence is bounded, we have proved (i).

(ii): Let $t \in \mathbb{R}$.

$$
\begin{aligned}
\left|\mathbb{E}\left(\mathrm{e}^{i t A_{n}}\right)-\mathbb{E}\left(\mathrm{e}^{i t B_{n}}\right)\right| & \leq|t| \mathbb{E}\left(\left|A_{n}-B_{n}\right|\right) \\
& \leq|t| \sum_{\ell=1}^{n}\left|\Xi_{f}\left(1 / \ell \delta_{n}\right)\right| \mathbb{E}\left(\left|a_{n, \ell}-W_{\ell}\right|\right),
\end{aligned}
$$

where, by [7, Lemma 4.4],

$$
\mathbb{E}\left(\left|a_{n, \ell}-W_{\ell}\right|\right) \leq \frac{C(\theta)}{n}+\frac{\theta}{n} \Psi_{n}(\ell) \ll_{\theta} \frac{1}{n}\left(1+\Psi_{n}(\ell)\right)
$$

for some $C(\theta)>0$ depending only on $\theta$ and for

$$
\Psi_{n}(\ell):=\prod_{k=0}^{\ell-1} \frac{n-k}{\theta+n-k-1} .
$$

Let $\left(u_{n}\right)_{n \geq 1}$ and $\left(v_{n}\right)_{n \geq 1}$ be two sequences of positive integers such that $u_{n}<v_{n}$ for all $n$ and also such that $u_{n}, \delta_{n}^{-1} / u_{n}, v_{n} /\left(\delta_{n}^{-1}\right)$ and $n / v_{n}$ all go to infinity with $n$. On 
the one hand,

$$
\begin{aligned}
& \frac{1}{n} \sum_{\substack{\ell<u_{n} \\
\text { or } \ell>v_{n}}}\left(1+\Psi_{n}(\ell)\right)\left|\Xi_{f}\left(1 / \ell \delta_{n}\right)\right| \\
& \quad \leq\left(\sup _{z \in\left(0, u_{n} \delta_{n}\right) \cup\left(v_{n} \delta_{n},+\infty\right)}\left|\Xi_{f}(1 / z)\right|\right) \frac{1}{n} \sum_{\ell=1}^{n}\left(1+\Psi_{n}(\ell)\right) \\
& \quad=\left(1+\frac{1}{\theta}\right) \sup _{z \in\left(0, u_{n} \delta_{n}\right) \cup\left(v_{n} \delta_{n},+\infty\right)}\left|\Xi_{f}(1 / z)\right| \\
& \quad \underset{n \rightarrow \infty}{\longrightarrow} 0,
\end{aligned}
$$

because $\Xi_{f}$ tends to zero at zero and at infinity, and by [7, Lemma 4.6],

$$
\frac{\theta}{n} \sum_{\ell=1}^{n} \Psi_{n}(\ell)=\sum_{\ell=1}^{n} \mathbb{P}\left[J_{n}=\ell\right]=1,
$$

where

$$
J_{n}=\min \left\{j \geq 1, \xi_{n-j+1}=1\right\}
$$

$\left(\xi_{j}\right)_{j \geq 1}$ being independent Bernoulli variables, $\xi_{j}$ having parameter $\theta /(\theta+j-1)$. On the other hand, noticing that $\Psi_{n}(\ell)$ is monotonic with respect to $\ell$ (the direction of monotonicity of $\Psi_{n}($.) changes at $\theta=1$ ) and tends to 1 when $n / \ell$ goes to infinity,

$$
\begin{aligned}
& \frac{1}{n} \sum_{u_{n} \leq \ell \leq v_{n}}\left(1+\Psi_{n}(\ell)\right)\left|\Xi_{f}\left(1 / \ell \delta_{n}\right)\right| \\
& \quad \leq\left\|\Xi_{f}\right\|_{\infty} \frac{v_{n}-u_{n}+1}{n} \times\left(1+\max \left(\Psi_{n}\left(u_{n}\right), \Psi_{n}\left(v_{n}\right)\right)\right) \\
& \quad \underset{n \rightarrow \infty}{\longrightarrow} 0,
\end{aligned}
$$

since $n / v_{n} \rightarrow \infty$ and then $\max \left(\Psi_{n}\left(u_{n}\right), \Psi_{n}\left(v_{n}\right)\right) \rightarrow 1$ as $n \rightarrow \infty$.

We can now deduce Theorem 1.1 (iii). Since $M^{\sigma_{n}}$ has $n$ eigenangles in each interval of length $2 \pi$, replacing $X_{\sigma_{n}, \delta_{n}}$ by $X_{\sigma_{n}, \delta_{n}}^{\prime}$ changes the sum by at most

$$
n \sum_{k \neq 0} \sup _{|x-2 k \pi| \leq \pi}\left|f\left(x / 2 \pi \delta_{n}\right)\right| \ll_{f} n \sum_{k \geq 1} \delta_{n}^{\alpha} k^{-\alpha} \ll_{f, \alpha} n \delta_{n}^{\alpha}
$$

quantity which, by the assumption made in (iii), tends to zero when $n \rightarrow \infty$. Using Slutsky's lemma, we easily deduce that (i) and (ii) are preserved when we replace $X_{\sigma_{n}, \delta_{n}}$ by $X_{\sigma_{n}, \delta_{n}}^{\prime}$. 


\section{Proof of Theorem 1.2}

We first show the following lemma:

Lemma 3 For all $n \geq 1$,

$$
\begin{aligned}
& \frac{1}{n} \sum_{j=1}^{n} \Psi_{n}(j)=\frac{1}{\theta}, \\
& \sum_{j=1}^{n} \frac{\Psi_{n}(j)}{j}=\sum_{j=1}^{n} \frac{1}{\theta+j-1},
\end{aligned}
$$

and

$$
\sum_{j=1}^{n} \frac{\Psi_{n}(j)}{j^{2}} \underset{n \rightarrow \infty}{\longrightarrow} \frac{\pi^{2}}{6}
$$

Proof The equalities (8) and (9) are proved in [6, Lemma 9]. Let us now show (10). Let $\left(u_{n}\right)_{n \geq 1}$ be a sequence of positive integers such that $u_{n}$ and $n / u_{n}$ both tend to infinity with $n$. We split the sum into two as follows

$$
\sum_{j=1}^{n} \frac{\Psi_{n}(j)}{j^{2}}=\sum_{j=1}^{u_{n}} \frac{\Psi_{n}(j)}{j^{2}}+\sum_{j=u_{n}+1}^{n} \frac{\Psi_{n}(j)}{j^{2}} .
$$

By monotonicity of $\Psi_{n}(k)$ with respect to $k$, we have

$$
\min _{k \leq u_{n}} \Psi_{n}(k)=\min \left(\Psi_{n}(1), \Psi_{n}\left(u_{n}\right)\right)
$$

and

$$
\max _{k \leq u_{n}} \Psi_{n}(k)=\max \left(\Psi_{n}(1), \Psi_{n}\left(u_{n}\right)\right)
$$

where $\lim _{n \rightarrow \infty} \Psi_{n}(1)=\lim _{n \rightarrow \infty} \Psi_{n}\left(u_{n}\right)=1$. Thus, as $n$ goes to infinity, we have

$$
\sum_{j=1}^{u_{n}} \frac{\Psi_{n}(j)}{j^{2}}=(1+o(1)) \sum_{j=1}^{u_{n}} \frac{1}{j^{2}}=\frac{\pi^{2}}{6}+o(1) .
$$

Besides,

$$
\sum_{j=u_{n}+1}^{n} \frac{\Psi_{n}(j)}{j^{2}} \leq \max _{k=u_{n}+1, \ldots, n} \Psi_{n}(k) \times \sum_{j=u_{n}+1}^{+\infty} \frac{1}{j^{2}}
$$




$$
\ll \theta \max \left(1, n^{1-\theta}\right) \times \frac{1}{u_{n}}
$$

which tends to 0 as $n$ goes to infinity if we take for instance $u_{n}:=\left\lfloor\max \left(n^{1-\frac{\theta}{2}}, n^{1 / 2}\right)\right\rfloor$.

We now have the ingredients needed in order to prove Theorem 1.2. The equality

$$
X_{\sigma_{n}, \delta_{n}}^{\prime}(f)-n \delta_{n} \hat{f}(0)=\sum_{\ell=1}^{n} a_{n, \ell} \Xi_{f, \ell}\left(\frac{1}{\ell \delta_{n}}\right)
$$

is proved similarly as (4), after taking into account the fact that $f(0)=0$. From Theorem 1.1, it is then enough to show

$$
\left|\mathbb{E}\left(\mathrm{e}^{i t A_{n}}\right)-\mathbb{E}\left(\mathrm{e}^{i t C_{n}}\right)\right| \underset{n \rightarrow \infty}{\longrightarrow} 0
$$

for all $t \in \mathbb{R}$, where $A_{n}:=\sum_{\ell=1}^{n} a_{n, \ell} \Xi_{f}\left(\frac{1}{\ell \delta_{n}}\right)$ and $C_{n}:=\sum_{\ell=1}^{n} a_{n, \ell} \Xi_{f, \ell}\left(\frac{1}{\ell \delta_{n}}\right)$. Let $t \in \mathbb{R}$.

$$
\begin{aligned}
\left|\mathbb{E}\left(\mathrm{e}^{i t A_{n}}\right)-\mathbb{E}\left(\mathrm{e}^{i t C_{n}}\right)\right| & \leq|t| \mathbb{E}\left(\left|A_{n}-C_{n}\right|\right) \\
& \leq|t| \sum_{\ell=1}^{n} \mathbb{E}\left(a_{n, \ell}\right)\left|\Xi_{f}\left(\frac{1}{\ell \delta_{n}}\right)-\Xi_{f, \ell}\left(\frac{1}{\ell \delta_{n}}\right)\right| \\
& =|t| \sum_{\ell=1}^{n} \frac{\theta \Psi_{n}(\ell)}{\ell}\left|\sum_{k=-\infty}^{\lfloor-\ell / 2\rfloor} f\left(\frac{k}{\ell \delta_{n}}\right)+\sum_{k=\lfloor\ell / 2\rfloor+1}^{+\infty} f\left(\frac{k}{\ell \delta_{n}}\right)\right| .
\end{aligned}
$$

Here, we use the fact that the expectation of the number of $\ell$-cycles is equal to $n / \ell$ times the probability that 1 is in an $\ell$-cycle, i.e., by the Feller coupling,

$$
\begin{aligned}
\mathbb{E}\left[a_{n, \ell}\right] & =\frac{n}{\ell} \mathbb{P}\left[\xi_{n}=\xi_{n-1}=\cdots=\xi_{n+2-\ell}=0, \xi_{n+1-\ell}=1\right] \\
& =\frac{n}{\ell} \frac{\theta}{n-\ell+\theta} \prod_{k=1}^{\ell-1} \frac{n-k}{n-k+\theta}=\frac{\theta \Psi_{n}(\ell)}{\ell}
\end{aligned}
$$

We now estimate the sum over the positive indices $\sum_{k=\lfloor\ell / 2\rfloor+1}^{+\infty} f\left(\frac{k}{\ell \delta_{n}}\right)$ : the sum over the negative indices behaves identically. To do this, we use the Euler-MacLaurin formula at order 2: for all positive integers $p<q$, and for all functions $g \in \mathcal{C}^{2}(\mathbb{R})$,

$$
\sum_{k=p}^{q} g(k)=\int_{p}^{q} g(x) \mathrm{d} x+\frac{g(p)+g(q)}{2}+\frac{g^{\prime}(q)-g^{\prime}(p)}{12}+\mathcal{O}\left(\int_{p}^{q}\left|g^{\prime \prime}(x)\right| \mathrm{d} x\right)
$$


so that if $g, g^{\prime}$ and $g^{\prime \prime}$ are integrable at $+\infty$, we have, letting $q$ tend to infinity,

$$
\sum_{k=p}^{+\infty} g(k)=\int_{p}^{+\infty} g(x) \mathrm{d} x+\frac{g(p)}{2}-\frac{g^{\prime}(p)}{12}+\mathcal{O}\left(\int_{p}^{+\infty}\left|g^{\prime \prime}(x)\right| \mathrm{d} x\right) .
$$

Applying this formula to $g(x)=f\left(\frac{x}{\ell \delta_{n}}\right)$ gives, with a change of variables into the integrals,

$\sum_{k=p}^{+\infty} f\left(\frac{k}{\ell \delta_{n}}\right)=-\ell \delta_{n} F\left(\frac{p}{\ell \delta_{n}}\right)+\frac{f\left(\frac{p}{\ell \delta_{n}}\right)}{2}-\frac{f^{\prime}\left(\frac{p}{\ell \delta_{n}}\right)}{12 \ell \delta_{n}}+\mathcal{O}\left(\frac{1}{\ell \delta_{n}} \int_{\frac{p}{\ell \delta_{n}}}^{+\infty}\left|f^{\prime \prime}(u)\right| \mathrm{d} u\right)$,

where $F$ is the antiderivative of $f$ such that $F(+\infty)=0$.

Then, with $p=\lfloor\ell / 2\rfloor+1$, using Taylor-Lagrange formula at order 3 on $F$, at order 2 on $f$ and at order 1 on $f^{\prime}$, between $\frac{1}{2 \delta_{n}}$ and $\frac{p}{\ell \delta_{n}}=\frac{1}{2 \delta_{n}}+\frac{1-\{\ell / 2\}}{\ell \delta_{n}}$, we get

$$
\begin{aligned}
\sum_{k=\lfloor\ell / 2\rfloor+1}^{+\infty} f\left(\frac{k}{\ell \delta_{n}}\right)= & -\ell \delta_{n} F\left(\frac{1}{2 \delta_{n}}\right)+\left[-(1-\{\ell / 2\})+\frac{1}{2}\right] f\left(\frac{1}{2 \delta_{n}}\right) \\
& +\frac{1}{\ell \delta_{n}}\left[-\frac{1}{2}(1-\{\ell / 2\})^{2}+\frac{1}{2}(1-\{\ell / 2\})-\frac{1}{12}\right] f^{\prime}\left(\frac{1}{2 \delta_{n}}\right) \\
& +\mathcal{O}\left(\frac{1}{\ell \delta_{n}} \int_{\frac{1}{2 \delta_{n}}}^{+\infty}\left|f^{\prime \prime}(u)\right| \mathrm{d} u\right) .
\end{aligned}
$$

Consequently,

$$
\begin{aligned}
\left|\sum_{k=\lfloor\ell / 2\rfloor+1}^{+\infty} f\left(\frac{k}{\ell \delta_{n}}\right)\right| \leq & \ell \delta_{n}\left|F\left(\frac{1}{2 \delta_{n}}\right)\right|+\frac{1}{2}\left|f\left(\frac{1}{2 \delta_{n}}\right)\right| \\
& +\frac{1}{12 \ell \delta_{n}}\left|f^{\prime}\left(\frac{1}{2 \delta_{n}}\right)\right| \\
& +\frac{1}{\ell} \times \mathcal{O}\left(\frac{1}{\delta_{n}} \int_{\frac{1}{2 \delta_{n}}}^{+\infty}\left|f^{\prime \prime}(u)\right| \mathrm{d} u\right),
\end{aligned}
$$

where we point out that the implicit constant involved in $\mathcal{O}$ does not depend on $\ell$. Finally, using (8), (9) and (10), it follows

$$
\begin{aligned}
\sum_{\ell=1}^{n} \frac{\theta \Psi_{n}(\ell)}{\ell}\left|\sum_{k=\lfloor\ell / 2\rfloor+1}^{+\infty} f\left(\frac{k}{\ell \delta_{n}}\right)\right| \leq & n \delta_{n}\left|F\left(\frac{1}{2 \delta_{n}}\right)\right| \\
& +\left(\frac{\theta}{2} \log n+\mathcal{O}_{\theta}(1)\right)\left|f\left(\frac{1}{2 \delta_{n}}\right)\right|
\end{aligned}
$$




$$
\begin{aligned}
& +\left(\frac{\theta \pi^{2}}{72}+o_{\theta}(1)\right) \frac{1}{\delta_{n}}\left|f^{\prime}\left(\frac{1}{2 \delta_{n}}\right)\right| \\
& +\mathcal{O}_{\theta}\left(\frac{1}{\delta_{n}} \int_{\frac{1}{2 \delta_{n}}}^{+\infty}\left|f^{\prime \prime}(u)\right| \mathrm{d} u\right),
\end{aligned}
$$

which tends to 0 as $n \rightarrow+\infty$, under the hypothesis made on $f$ and $\delta_{n}$.

Open Access This article is licensed under a Creative Commons Attribution 4.0 International License, which permits use, sharing, adaptation, distribution and reproduction in any medium or format, as long as you give appropriate credit to the original author(s) and the source, provide a link to the Creative Commons licence, and indicate if changes were made. The images or other third party material in this article are included in the article's Creative Commons licence, unless indicated otherwise in a credit line to the material. If material is not included in the article's Creative Commons licence and your intended use is not permitted by statutory regulation or exceeds the permitted use, you will need to obtain permission directly from the copyright holder. To view a copy of this licence, visit http://creativecommons.org/licenses/by/4.0/.

\section{References}

1. Arratia, R.: On the central role of scale invariant Poisson processes on $(0, \infty)$. In: Microsurveys in Discrete Probability (Princeton, NJ, 1997), vol 41 of DIMACS Ser. Discrete Math. Theoret. Comput. Sci., pp. 21-41. American Mathematical Society, Providence, RI (1998)

2. Arratia, R., Barbour, A.D., Tavaré, S.: Logarithmic combinatorial structures: a probabilistic approach. EMS Monographs in Mathematics. European Mathematical Society (EMS), Zürich (2003)

3. Arratia, R., Barbour, A.D., Tavaré, S.: A tale of three couplings: Poisson-Dirichlet and GEM approximations for random permutations. Comb. Probab. Comput. 15(1-2), 31-62 (2006)

4. Bahier, V.: Characteristic polynomials of modified permutation matrices at microscopic scale. Stoch. Proces. Their Appl. 129(11), 4335-4365 (2018)

5. Bahier, V.: On a limiting point process related to modified permutation matrices. arXiv preprint arXiv:1803.03546 (2018)

6. Bahier, Valentin: On the number of eigenvalues of modified permutation matrices in mesoscopic intervals. J. Theor. Probab. 32(2), 974-1022 (2019)

7. Ben Arous, G., Dang, K.: On fluctuations of eigenvalues of random permutation matrices. Ann. Inst. Henri Poincaré Probab. Stat. 51(2), 620-647 (2015)

8. Berggren, T., Duits, M.: Mesoscopic fluctuations for the thinned circular unitary ensemble. Math. Phys. Anal. Geom. 20(3), 40 (2017)

9. Bourgade, P.: Mesoscopic fluctuations of the zeta zeros. Probab. Theory Relat Fields 148(3-4), 479-500 (2010)

10. Chhaibi, R., Najnudel, J., Nikeghbali, A.: The circular unitary ensemble and the Riemann zeta function: the microscopic landscape and a new approach to ratios. Invent. Math. 207(1), 23-113 (2017)

11. Costin, O., Lebowitz, J.L.: Gaussian fluctuation in random matrices. Phys. Rev. Lett. 75(1), 69-72 (1995)

12. Dang, K.: The spectrum of random permutation matrices. $\mathrm{PhD}$ thesis, University of Zurich (2012)

13. Diaconis, P., Evans, S.N.: Linear functionals of eigenvalues of random matrices. Trans. Am. Math. Soc. 353(7), 2615-2633 (2001)

14. Diaconis, P., Shahshahani, M.: On the eigenvalues of random matrices. J. Appl. Probab. 31(31), 49-62 (1994)

15. Duits, M., Johansson, K.: On Mesoscopic Equilibrium For Linear Statistics in Dyson's Brownian Motion. American Mathematical Society, Providence (2018)

16. Evans, S.N.: Eigenvalues of random wreath products. Electron. J. Probab. 7(9), 15 (2002)

17. Ewens, W.J.: The sampling theory of selectively neutral alleles. Theor. Popul. Biol. 3, 87-112 (1972)

18. Feller, W.: The fundamental limit theorems in probability. Bull. Am. Math. Soc. 51, 800-832 (1945) 
19. He, Yukun: Mesoscopic linear statistics of Wigner matrices of mixed symmetry class. J. Stat. Phys. 175(5), 932-959 (2019)

20. He, Y., Knowles, A.: Mesoscopic eigenvalue statistics of Wigner matrices. Ann. Appl. Probab. 27(3), $1510-1550$ (2017)

21. Hughes, C., Najnudel, J., Nikeghbali, A., Zeindler, D.: Random permutation matrices under the generalized Ewens measure. Ann. Appl. Probab. 23(3), 987-1024 (2013)

22. Johansson, K.: On random matrices from the compact classical groups. Ann. Math. 145, 519-545 (1997)

23. Johansson, K., Lambert, G.: Gaussian and non-Gaussian fluctuations for mesoscopic linear statistics in determinantal processes. Ann. Probab. 46(3), 1201-1278 (2018)

24. Lambert, G.: Mesoscopic fluctuations for unitary invariant ensembles. Electron. J. Probab. 23, 7-33 (2018)

25. Lambert, G.: Mesoscopic central limit theorem for the circular beta-ensembles and applications. arXiv preprint arXiv:1902.06611 (2019)

26. Lambert, G., Ostrovsky, D., Simm, N.: Subcritical multiplicative chaos for regularized counting statistics from random matrix theory. Commun. Math. Phys. 360(1), 1-54 (2018)

27. Manstavichyus, Ė.: The Poisson distribution for linear statistics of random permutations. Liet. Mat. Rink. 45(4), 537-552 (2005)

28. Najnudel, J., Nikeghbali, A.: The distribution of eigenvalues of randomized permutation matrices. Ann. Inst. Fourier (Grenoble) 63(3), 773-838 (2013)

29. Soshnikov, A.: The central limit theorem for local linear statistics in classical compact groups and related combinatorial identities. Ann. Probab. 28(3), 1353-1370 (2000)

30. Soshnikov, A.B.: Gaussian fluctuation for the number of Particles in airy, bessel, sine, and other determinantal random point fields. J. Stat. Phys. 100(3/4), 491-522 (2000)

31. Tsou, B.: Eigenvalue fluctuations of symmetric group permutation representations on k-tuples and k-subsets. arXiv preprint arXiv:1810.11904 (2018)

32. Tsou, B.: The eigenvalue point process for symmetric group permutation representations on k-tuples. arXiv preprint arXiv:1901.06721 (2019)

33. Wieand, K.: Eigenvalue distributions of random unitary matrices. Probab. Theory Related Fields 123(2), 202-224 (2002)

34. Wieand, Kelly: Eigenvalue distributions of random permutation matrices. Ann. Probab. 28(4), 15631587 (2000)

35. Wieand, K.: Permutation matrices, wreath products, and the distribution of eigenvalues. J. Theoret. Probab. 16(3), 599-623 (2003)

Publisher's Note Springer Nature remains neutral with regard to jurisdictional claims in published maps and institutional affiliations. 